\title{
UTILIZAÇÃO DO RETENTADO DA ULTRAFILTRAÇÃO DO SUCO DE ACEROLA NA ELABORAÇÃO DE LICOR
}

\author{
EDMAR DAS MERCÊS PENHA * \\ NATASCHA C. A. S. BRAGA ** \\ VIRGIINIA MARTINS DA MATTA * \\ LOURDES MARIA C. CABRAL * \\ REGINA CÉLIA DELLA MODESTA * \\ SIDINÉIA CORDEIRO DE FREITAS *
}

\begin{abstract}
Estudou-se a preparação de licor mediante maceração alcoólica da fração retida no processo de ultrafiltração do suco de acerola, em sistema de membranas tubulares com peso molecular de corte de $30-80 \mathrm{kDa}$, área filtrante de $0,05 \mathrm{~m}^{2}$ e pressão de $200 \mathrm{kPa}$. As características sensoriais e nutricionais do licor foram avaliadas tendo-se como referência o licor preparado a partir da polpa de acerola fresca pelo mesmo processo de fabricação. Os licores foram preparados partindo-se de mistura 1:1 de polpa de acerola ou do retentado da ultrafiltração e álcool de milho. Após 15 dias de maceração adicionou-se açúcar até concentração de $250 \mathrm{~g} / \mathrm{L}$ e o teor alcoólico foi ajustado para $18^{\circ} \mathrm{GL}$. Os licores apresentaram teores de vitamina $\mathrm{C}$ semelhantes, na faixa de $0,400 \mathrm{~g} / 100 \mathrm{~g}$, cerca de seis vezes mais que a dose diária recomendada para um adulto. As análises microbiológicas da polpa de acerola, do retentado e dos licores atenderam aos padrões estabelecidos pela legislação vigente. $\mathrm{O}$ aroma alcoólico tornou-se mais evidenciado no licor de retentado enquanto que o aroma e o sabor frutal mostraram-se mais pronunciados no licor de polpa de acerola. A percepção do aroma doce também foi bastante pronunciada no licor de polpa de acerola. A pungência do licor de retentado mostrou-se menor que a do licor de polpa de acerola. Os resultados obtidos demonstraram que o licor elaborado a partir do retentado da ultrafiltração do suco de acerola apresenta características similares às do licor preparado com a própria polpa.
\end{abstract}

PALAVRAS-CHAVE: ACEROLA; LICOR; BEBIDAS ALCOÓLICAS; SUCO DE FRUTA.

* Pesquisadores, Embrapa Agroindústria de Alimentos, Rio de Janeiro, RJ. (e-mail: epenha@radnet.com.br).

** Bolsista, Pontifícia Universidade Católica do Rio de Janeiro (PUC), Rio de Janeiro, RJ. 


\section{INTRODUÇÃO}

A acerola apresenta teor de vitamina $C$ na faixa de 1.000 a $4.000 \mathrm{mg} /$ $100 \mathrm{~g}$ de polpa dependendo do estágio de maturação da fruta, clima e localização do plantio, sendo maior quando o fruto ainda se encontra verde (ASENJO \& GUZMAN, 1946). Em comparação com a laranja, a acerola apresenta cerca de 90 vezes mais vitamina $C$ e duas vezes mais magnésio, potássio e ácido pantotênico. Estão também presentes no fruto, vitamina $\mathrm{A}(4,3 \mathrm{a} 12,5 \mathrm{Ul} / 100 \mathrm{~g})$, tiamina, riboflavina e niacina, em concentrações comparáveis com as de outras frutas (RAINTREE GROUP, 1998).

Apesar da qualidade nutricional do fruto, o processamento da acerola tem se restringido basicamente à produção de polpa, conservada sob congelamento, após pasteurização ou não. Os processamentos que envolvem o uso do calor geralmente promovem alterações significativas nos atributos sensoriais e nutricionais das frutas. As modificações bioquímicas decorrentes do processamento da acerola para a produção de sucos estão relacionadas a fatores como: a presença de substâncias pécticas que conferem turbidez indesejada ao produto; as reações dos pigmentos carotenóides e antociânicos, responsáveis pela coloração da acerola, um de seus principais atributos sensoriais; e a perda do teor vitamínico pela degradação dos ácidos ascórbico e dehidroascórbico e dos carotenos (BOBBIO \& BOBBIO, 1992).

A maioria das reações degradativas é oxidativa e depende de fatores como tensão de oxigênio, temperatura, luminosidade, acidez, presença de metais e atividade da água (BOBBIO \& BOBBIO, 1992). O oxigênio é o principal responsável pela degradação das antocianinas do suco de acerola (ROCHA, 1988). No entanto, pode ocorrer também a degradação enzimática pela ação de oxidases. A oxidação resulta na isomerização de carotenos da forma trans para a cis ocasionando a perda do poder vitamínico (GODOY \& RODRIGUES-AMAYA, 1994). A degradação da vitamina $C$ pode também ocorrer pela ação de enzimas oxidativas encontradas na própria fruta como a citocromo oxidase, a ácido ascórbico oxidase e a peroxidase (NAGY, 1980; OLIVA, 1995).

O tratamento térmico pode inibir as reações enzimáticas no suco de acerola. Entretanto, a pasteurização do suco de acerola integral, realizada em trocador de placas a $90{ }^{\circ} \mathrm{C}$ por 45 segundos, por exemplo, provoca perda de vitamina C de 12\% (MATSUURA, 1994). 
O processo de ultrafiltração tem se mostrado como excelente alternativa para a elaboração de sucos clarificados. A ultrafiltração consiste na filtração, através de membranas semipermeáveis, com capacidade para reter bactérias, emulsão água-óleo, polímeros, pigmentos, vírus, proteínas e polissacarídios. A fração que não permeia a membrana (poros menores que o tamanho das moléculas), denominada retentado, é o resíduo do processo de clarificação.

A clarificação do suco de acerola por ultrafiltração preserva as propriedades nutricionais e sensoriais da fruta e reduz as reações degradativas decorrentes da ação de enzimas constitutivas do próprio fruto (MENSAH \& LOZANO, 1992; ITOUA GASSAYE et al., 1991). Entretanto, a grande quantidade de retentado, gerada neste processo, pode constituir-se em entrave para a aplicação desta tecnologia em escala industrial, devido ao impacto do seu descarte no meio ambiente. O retentado, por sua composição similar à do suco de acerola, pode ser aproveitado como matéria-prima para a elaboração de diferentes produtos, como licor, por exemplo.

O licor é uma mistura de água e álcool potáveis, açucarada, adicionada de certa substância, com predomínio aromático e diversos constituintes em proporções determinadas que podem apresentar valor medicinal (TRITTON, 1975).

Neste trabalho foi estudada a preparação de licor mediante maceração alcoólica do retentado da ultrafiltração do suco de acerola. O licor de retentado foi comparado, em suas características sensoriais e nutricionais, ao licor preparado a partir da polpa de acerola fresca, utilizando-se o mesmo processo de fabricação.

\section{MATERIAL E MÉTODOS}

Utilizou-se polpa de acerola (Malpighia glabra L.) congelada comercial, não-pasteurizada, para a obtenção do suco clarificado e do licor tradicional. Para a elaboração do licor de retentado, a matéria-prima utilizada foi a fração retida pelas membranas no processo de clarificação da polpa de acerola por ultrafiltração. Os demais ingredientes empregados no preparo dos licores foram: açúcar cristalizado comercial, álcool de milho a $96,5^{\circ} \mathrm{GL}$ e água destilada. A polpa de acerola foi descongelada até a temperatura ambiente $\left(28^{\circ} \mathrm{C}\right)$ em vaso multipropósito (ARMFIELD), com capacidade para $15 \mathrm{~L}$, encamisado e com agitação. A seguir, a polpa foi 
ultrafiltrada em sistemas de membranas tubulares de polisulfona $(\mathrm{KOCH}$ MEMBRANE SYSTEMS), com peso molecular de corte de 30-80 kDa, área filtrante de $0,05 \mathrm{~m}^{2}$ e pressão de $200 \mathrm{kPa}$. A fração retida foi, imediatamente, congelada e armazenada $\mathrm{a}-10^{\circ} \mathrm{C}$ até a sua utilização.

Os licores foram preparados após descongelamento da polpa de acerola ou do retentado da ultrafiltração, efetuando-se a mistura 1:1 com álcool de milho. Após 15 dias de maceração adicionou-se açúcar até concentração de $250 \mathrm{~g} / \mathrm{L}$, sendo o teor alcoólico ajustado para $18^{\circ} \mathrm{GL}$. O cálculo do teor de álcool e de açúcar foi realizado utilizando-se o Quadrado de Pierson, citado por TANADA (1996). Foram realizadas três repetições de cada experimento em duplicata.

O controle das características físico-químicas foi realizado pelas determinações de $\mathrm{pH}$, densidade, viscosidade aparente, sólidos solúveis, acidez titulável e teor alcoólico (ASSOCIATION OF OFFICIAL ANALYTICAL CHEMISTS, 2000), vitamina C (POLESELLO \& RIZZOLO, 1990), antocianinas (MARKAKIS, 1982) e carotenóides totais (TAKAHASHI, 1987).

Para a determinação dos principais atributos sensoriais de qualidade dos licores foram selecionados e treinados cinco provadores, bem como definidos os seguintes atributos: aderência (formação de colar nas paredes de um copo); aroma alcoólico, frutal e doce; acidez, maciez (suavidade); pungência (dormência na boca); viscosidade (fluidez na boca); sabor alcoólico, sabor frutal e gosto doce. Empregou-se o método de Análise Descritiva Quantitativa - ADQ (STONE et al., 1974) utilizando-se escala não-estruturada de $10 \mathrm{~cm}$ (valor 1 correspondente a fraco e valor 9 a forte). Para cada atributo os valores foram obtidos medindo-se a distância da extremidade esquerda da escala (zero) até a marca assinalada pelo provador (MEILGAARD et al., 1991).

A caracterização microbiológica da polpa de acerola e do retentado da ultrafiltração da polpa de acerola foi realizada mediante pesquisa de Salmonella sp. e de coliformes a $45^{\circ} \mathrm{C}$ (VANDERZANT \& SPLITTSTOESSER, 1992).

\section{RESULTADOS E DISCUSSÃO}

No processo de ultrafiltração da polpa de acerola, a fração retida representou cerca de $60 \%$ do volume da polpa original. O baixo rendimento em suco clarificado (30\%) pode ser atribuído à pequena área filtrante 
empregada no processo $\left(0,05 \mathrm{~m}^{2}\right)$. Este resultado justifica ainda mais a procura por alternativas de utilização da fração retida visando agregar valor à mesma.

O retentado apresentou valores de $\mathrm{pH}$, densidade e teor de vitamina $\mathrm{C}$ próximos aos encontrados para a polpa de acerola congelada (Tabela 1). A acidez e os teores de sólidos solúveis e de carotenóides, porém, foram maiores no retentado da ultrafiltração enquanto que a viscosidade aparente e o teor de antocianinas, menores. É importante ressaltar que as características do retentado da ultrafiltração mostraram-se bem próximas às da polpa (MATTA, 1999).

As variações encontradas entre as características físico-químicas do retentado e da polpa, utilizadas para o licor-referência, devem-se ao fato dos mesmos serem provenientes de diferentes lotes de acerola. As características da fruta variam em função do clima e condições de cultivo, do período de colheita, variedade e maturidade, entre outros. Pôde ser observado, portanto, que as pequenas diferenças de composição nas matérias-primas refletiram-se nos respectivos licores. Considerando os valores das médias e desvios padrão dos teores de vitamina $C$ verifica-se que não houve diferença entre os dois licores. $O$ teor de vitamina $C$ do licor de retentado $(0,390 \mathrm{mg} / 100 \mathrm{~g})$ mostrou-se cerca de seis vezes maior que a dose diária recomendada para um adulto, ou seja, 0,070 mg/100 g (MARINO NETTO, 1986).

As contagens de Salmonella sp. (ausência em $25 \mathrm{~g}$ ) e de coliformes a $45^{\circ} \mathrm{C}(<3 \mathrm{NMP} / \mathrm{g})$ da polpa de acerola, do retentado e dos respectivos licores atenderam aos padrões estabelecidos pela legislação brasileira para sucos de frutas consumidos in natura e bebidas alcoólicas (ANVISA, 2001).

O gráfico obtido na análise descritiva quantitativa (Figura 1) demonstra que não houve diferença significativa entre os principais atributos dos dois licores avaliados. No entanto, o aroma alcoólico tornou-se mais evidenciado no licor de retentado devido, provavelmente, à perda de substâncias aromáticas da polpa de acerola durante o processo de obtenção do suco clarificado. Este fato pode ser confirmado pelo aroma e sabor frutal mais pronunciado verificado no licor de polpa de acerola que no licor de retentado. A percepção do aroma doce também foi mais acentuada no licor de polpa de acerola. Embora não tenha havido diferença significativa entre a acidez e o sabor alcoólico dos dois licores, a pungência do licor de retentado mostrou-se significativamente menor que a do licor de polpa de acerola. 

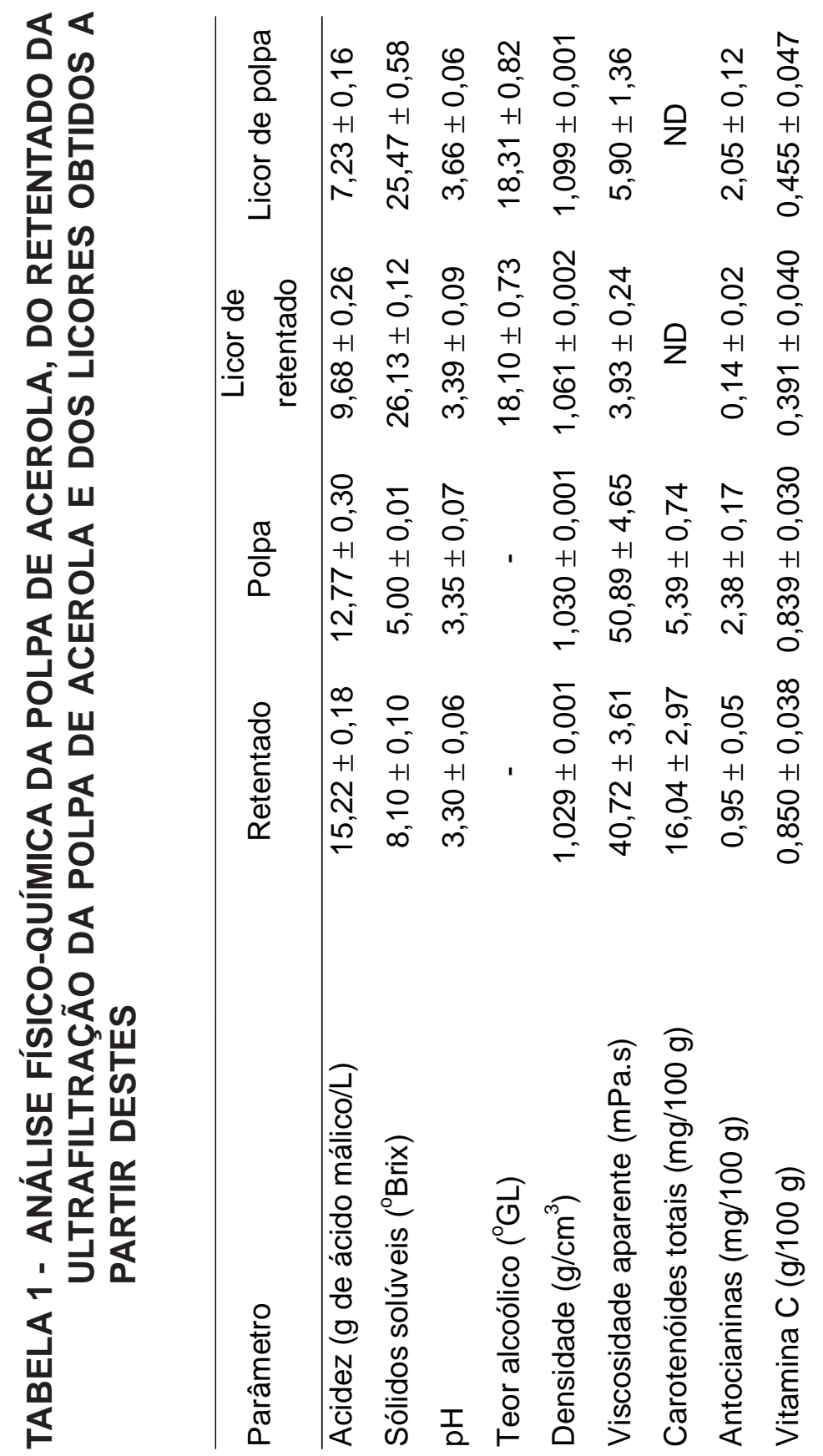

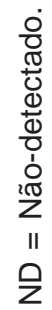




\section{FIGURA1- CONFIGURAÇÃO DA ANÁLISE DESCRITIVA QUANTITATIVA DOS LICORES DE ACEROLA E DE RETENTADO}

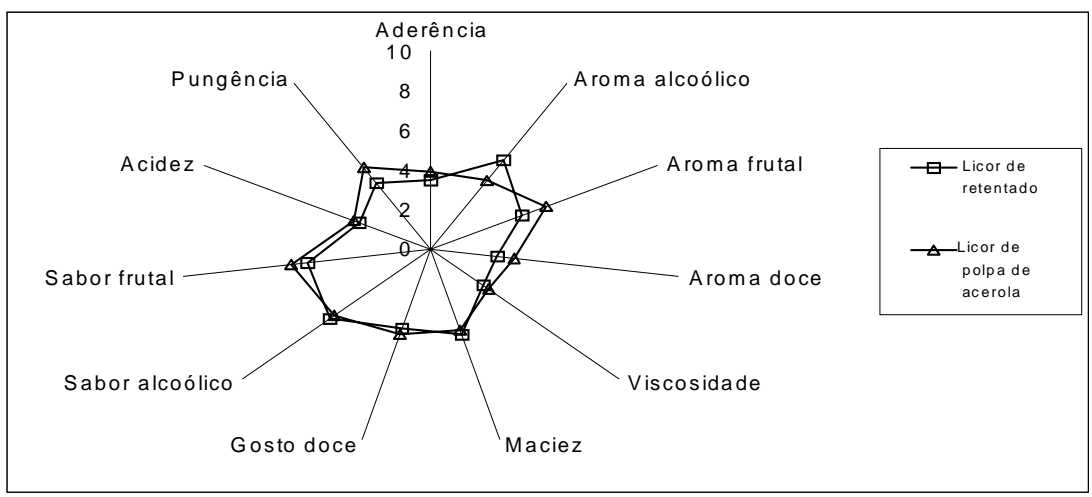

\section{CONCLUSÃO}

Os resultados obtidos demonstram que o licor elaborado a partir do retentado da ultrafiltração do suco de acerola apresenta características similares àquelas do licor preparado a partir da própria polpa. O teor de vitamina $C$ do licor de retentado $(0,390 \mathrm{mg} / 100 \mathrm{~g})$ caracteriza-o como produto com alto valor nutricional. A obtenção deste produto representa alternativa para utilização de subproduto do processo de clarificação do suco de acerola que, além de agregar valor, minimiza a geração de resíduo orgânico e, conseqüentemente, o custo para descarte deste resíduo.

\section{Abstract \\ UTILIZATION OF ACEROLA JUICE ULTRAFILTRATION RETENTATE IN A LIQUEUR ELABORATION}

The preparation of a liqueur by alcoholic maceration of the fraction retained in the acerola juice ultrafiltration process, in tubular membrane system with molecular weight of $30-80 \mathrm{kDa}$ cut-off, filtration area of $0.05 \mathrm{~m}^{2}$ and $200 \mathrm{kPa}$ of transmembrane pressure was studied. The nutritional and sensory characteristics of the liqueur were evaluated having as standard the liqueur prepared from fresh acerola pulp by the same fabrication process. Liqueurs were prepared from a 1:1 mixture of acerola pulp or retentate and corn alcohol. After 15 days of maceration, sugar was added until a concentration of $250 \mathrm{~g} / \mathrm{L}$ and alcohol grade was adjusted to $18^{\circ} \mathrm{GL}$. The vitamin $\mathrm{C}$ content of both liqueurs 
were similar, about $0,400 \mathrm{~g} / 100 \mathrm{~g}$, corresponding to almost six times the vitamin $\mathrm{C}$ recommended dietary allowance for an adult. Microbiological analyses of acerola pulp, retentate and liqueurs attended the standards established by legislation. Alcoholic aroma was more pronounced in the retentate liqueur while the fruit aroma and flavor were more pronounced in the pulp liqueur. The perception of the sweet aroma was also well pronounced in the acerola pulp liqueur. The pungency of the retentate liqueur was lower than the acerola pulp liqueur. The results obtained showed that the liqueur elaborated from the acerola juice ultrafiltration retentate present similar characteristics to the liqueur prepared from the pulp.

KEY-WORDS: ACEROLA; LIQUEUR; ALCOHOLIC BEVERAGES; FRUIT JUICE.

\section{REFERÊNCIAS}

1 ANVISA. Agência Nacional de Vigilância Sanitária. Resolução RDC n.12, de 2 de janeiro de 2001. Aprova o regulamento técnico sobre padrões microbiológicos para alimentos. Disponível em: <http:/ /anvisa.gov.br/legi/resol/index_2001_rdc.htm>. Acesso em: 02 ago.2001.

2 ASENJO, C.F.; DE GUZMAN, A.R.F. The high ascorbic acid content of the West Indian cherry. Science, v.103, n.2669, p.219, 1946.

3 ASSOCIATION OF OFFICIAL ANALYTICAL CHEMISTS. Official methods of analysis of AOAC. $17^{\text {th }}$ ed. Gaithersburg, 2000. v. 2

4 BOBBIO, F.O.; BOBBIO, P.A. Introdução à química de alimentos. 2. ed. São Paulo: Varela, 1992. 223 p.

5 GODOY, H.T.; RODRIGUES-AMAYA, D.B. Occurrence of cis-isomers of provitamin A in brazilian fruits. Journal of Agricultural and Food Chemistry, v.42, n.6, p.1306-1313, 1994.

6 ITOUA GASSAYE, S.; DAVIN, A.; PEUCHOT, M.; BENAIM, R. Intérêt des techniques à membrane dans la production de jus de fruits tropicaux (cas des jus clarifiés d'ananas): I'evolution des flux de filtration an cours du procédé. Fruits, v.46, n.3, p.251-258, 1991.

7 MARINO NETTO, L. Acerola: a cereja tropical. São Paulo: Nobel, 1986. $94 \mathrm{p}$.

8 MARKAKIS, P. Anthocyanins as food colors. London: Academic Press, 1982. $263 \mathrm{p}$. 
9 MATSUURA, F.C.A.U. Processamento e caracterização de suco integral e concentrado congelado de acerola. Campinas, 1994. 141 f. Dissertação (Mestrado em Tecnologia de Alimentos) Faculdade de Engenharia de Alimentos, Universidade Estadual de Campinas.

10 MATTA, V.M. Estudo da utilização dos processos de separação por membranas para obtenção de suco de acerola clarificado e concentrado. Campinas, 1999. $181 \mathrm{f}$. Tese (Doutorado em Tecnologia de Alimentos) - Faculdade de Engenharia de Alimentos, Universidade Estadual de Campinas.

11 MEILGAARD, M.; CIVILLE, G.V.; CARR, B.T. Sensory evaluation techniques. $2^{\text {nd }} e d$. Florida: CRC Press, 1991. $354 \mathrm{p}$.

12 MENSAH, A.; LOZANO, Y. Application de la microfiltration en flux tangentiel dans la fabrication de concentrés citriques. Agrumes, Numeró Spécial, p.271-272, 1992.

13 NAGY, S. Vitamin C content of citrus fruit and their products. A review. Journal of Agricultural and Food Chemistry, n.28, p.8-18, 1980.

14 OLIVA, P. B. Estudo do armazenamento da acerola in natura e estabilidade do néctar de acerola. Campinas, 1995. $103 \mathrm{f}$. Dissertação (Mestrado em Tecnologia de Alimentos) - Faculdade de Engenharia de Alimentos, Universidade Estadual de Campinas.

15 POlesello, A.; RIZZOLO, A. Application of HPLC to the determination of water-soluble vitamins in foods: 2 . A review, (19859). Journal of Micronutrient Analysis, v. 8, p. 105-158, 1990.

16 RAINTREE GROUP. Acerola. Disponível em: <http://www.raintree.com/acerola. htm>. Acesso em: 6 ago. 1998.

17 ROCHA, I.C. Suco de acerola: efeito da temperatura de pasteurização e armazenamento. Recife, 1988. 105 f. Dissertação (Mestrado) - Centro de Ciências da Saúde, Universidade Federal de Pernambuco.

18 STONE, H.; SIEDEL, J.; OLIVER, S. Sensory evaluation by quantitative descriptive analysis. Food Technology, v.28, n.11, p.2434, 1974. 
19 TAKAHASHI, M.Y. Monografia de corantes naturais para fins alimentícios: padrões de qualidade e identidade. 2. ed. São Paulo: Instituto Adolfo Lutz, 1987. $118 \mathrm{p}$.

20 TANADA, P.S. Obtenção de extrato de banana (Musa cavendishii) isento de polifenol oxidase por ultrafiltração e concentrado por osmose inversa. Campinas, 1996. 101 f. Dissertação (Mestrado em Tecnologia de Alimentos) - Faculdade de Engenharia de Alimentos, Universidade Estadual de Campinas.

21 TRITTON, S.M. Spirits, aperitifs and liqueurs: their production. London: Faber and Faber, 1975. $82 \mathrm{p}$.

22 VANDERZANT, C.; SPLITTSTOESSER, D.F. Compendium of methods for the microbiological examination of foods. $3^{\text {rd }} \mathrm{ed}$. Washington, D.C: APHA, 1992. Cap. 16, 24, 25. 